\title{
COLOR IMAGE WATERMARKING USING THE SPATIO-CHROMATIC FOURIER TRANSFORM
}

\author{
Tsz Kin Tsui, Xiao-Ping Zhang, Dimitri Androutsos \\ Ryerson University, Department of Electrical and Computer Engineering \\ 350 Victoria Street, Toronto, Ontario, Canada \\ \{ttsui,xzhang,dimitri\}@ee.ryerson.ca
}

\begin{abstract}
In this paper, a color watermarking algorithm is proposed. The method encodes the chromatic content of a color image as CIE $a^{*} b^{*}$ chromaticity coordinates whereas the achromatic content is encoded as CIE $L$ tristimulus value. Color watermarks (yellow and blue) are embedded in the frequency domain of the chromatic channels by using the Spatio Chromatic Discrete Fourier Transform (SCDFT). It first encodes $a^{*}$ and $b^{*}$ as complex values, followed by a single discrete Fourier Transform. Watermark casting is performed by estimating the Just-Noticeable distortion (JND) of the images, to ensure watermark invisibility. The most interesting characteristics of the new scheme is the possibility of performing watermarking in the frequency domain of chromatic components.
\end{abstract}

\section{INTRODUCTION}

Digital image watermarking is the communication of information by embedding additional information in images, without introducing any perceptual change. Nowadays it can used for copyright protection, content authentication, and owner identification. However, most existing color watermarking algorithms mark the channels of images separately [1] [2], which does not mimic the operation of the human visual system (HVS). To overcome this problem, researchers have proposed models to process the color channels intrinsically. For example, Reed $e t$. al. proposed a system that takes advantage of the low sensitivity of the human visual system to high frequency changes along the yellowblue axis, to place most of the watermark in the yellow component of the image [3]. Bas et. al. proposed a digital color image watermarking scheme using the hypercomplex numbers representation and the Quaternion Fourier Transform [4].

This paper introduces a new color watermarking algorithm that uses the Spatio-Chromatic Discrete Fourier Transform (SCDFT) for data embedding. Of the particular relevance is the theory of "Spatio-chromatic image encoding and feature extraction" proposed by McCabe [5], it shows the interpretation of the spectral domain information and demonstrates how points in the frequency domain represent sinusoidally varying chromatic information in the spatial domain.

This paper is organized as follows: First, the theory of spatio-chromatic image processing is discussed. Then, a new watermarking scheme using the SCDFT is presented and evaluated. Finally our future work is also discussed.

\section{SPATIO-CHROMATIC IMAGE PROCESSING}

Spatio-chromatic image processing encodes only the color information of an image (for example, $a^{*}$ and $b^{*}$ channels in the $L a^{*} b^{*}$ color space) to complex numbers $a+j b$, and analyze it via one complex frequency transform - Spatiochromatic Discrete Fourier Transform (SCDFT) [5], which is defined as follows:

$$
\begin{aligned}
& A(P, Q)+j B(P, Q)= \\
& \sum_{p=-\frac{N}{2}+1}^{\frac{N}{2}} \sum_{q=-\frac{N}{2}+1}^{\frac{N}{2}}\left[a^{*}(p, q)+j b^{*}(p, q)\right] * e^{\left.\frac{(-2 \pi j(P p+Q q)}{N}\right)}
\end{aligned}
$$

where $N \times N$ is the size of the image, $(p, q)$ are the image spatial coordinates, $(P, Q)$ are the frequency coordinates, and $(A, B)$ are the real and imaginary values occurring at the spatial frequency $(P, Q)$, which determines how the colors in the image changes spatially. The concept of phasors gives an interpretation that each pair of frequency values results in rainbow gratings of different frequency and orientation in the image, which are combined to form the color image [5]. McCabe also pointed out that it is possible to generate spatial frequencies with modulations operating in either the red-green or yellow-blue color opponency paths in the color space, if $A(P, Q), B(P, Q)$, $A(-P,-Q), B(-P,-Q)$ satisfy the following conditions: 
- For variation along red-green, $A(P, Q)=k, B(P, Q)=$ $0, A(-P,-Q)=k, B(-P,-Q)=0$, such that the resultant path only varies along the real axis

- For variation along yellow-blue, $A(P, Q)=0, B(P, Q)=$ $k, A(-P,-Q)=0, B(-P,-Q)=k$, which makes the resultant path varies along the imaginary axis.

\section{NEW WATERMARK EMBEDDING SCHEME}

\subsection{Color Spaces}

The CIE $L a^{*} b^{*}$ color model proposed in 1976 is the chromaticity model chosen for this paper [7]. The underlying reason is that it is a uniform color space used to measure the color difference $\triangle E$ at each pixel, which is defined as:

$$
\triangle E=\sqrt{\triangle L^{2}+\left(\triangle a^{*}\right)^{2}+\left(\triangle b^{*}\right)^{2}} .
$$

Experiments have shown that $\triangle E<1$ is not detectable by humans, and $\triangle E<3$ is not apparent. In C.H. Chou's paper [6], $\triangle E=3$ is called Uniform Just-Noticeable Color Difference (UJNCD), and he derived a JND model, which he called Non-Uniform Just-Noticeable Color Difference (NU$J N C D$ ) after considering some masking effects. This paper uses his model to bound the distortion generated by embedding the watermarks.

\subsection{Human Visual System}

A good model of the HVS should be used to ensure watermark invisibility. The importance of the HVS cannot be overemphasized and much research exists on its characteristics and behavior [8]. This paper uses the following two characteristics of the HVS to satisfy the invisibility requirement: 1) The eye is particularly sensitive to changes in image hue; and 2) the eye is less sensitive to yellow-blue component [9]. The next section will demonstrate how to satisfy the requirements in the SCDFT domain.

\subsection{New Adaptive Color Watermarking Algorithm}

Given an image of size $N \times N$, we divide the image into blocks of size $8 \times 8$, following by taking the SCDFT of each block. We embed a pair of watermarks $w\left(k_{1}, k_{2}\right)$ and $w\left(-k_{1},-k_{2}\right)$ into the coefficients $f\left(k_{1}, k_{2}\right)$ and $f\left(-k_{1},-k_{2}\right)$ in the following way:

$$
\begin{aligned}
& f^{\prime}\left(k_{1}, k_{2}\right)=f\left(k_{1}, k_{2}\right) \pm \alpha w\left(k_{1}, k_{2}\right) \\
& \quad f^{\prime}\left(-k_{1},-k_{2}\right)=f\left(-k_{1},-k_{2}\right) \pm \alpha w\left(-k_{1},-k_{2}\right),
\end{aligned}
$$

where $\alpha$ is the watermark strength to be maximized. To apply the two characteristics of the HVS, some constraints are imposed to the values $w\left(k_{1}, k_{2}\right)$ and $w\left(-k_{1},-k_{2}\right)$. Let $f\left(k_{1}, k_{2}\right)=f_{r p}+j f_{i p}, w\left(k_{1}, k_{2}\right)=w_{r p}+j w_{i p}$, and $w\left(-k_{1},-k_{2}\right)=w_{r n}+j w_{i n}$, where the subscript $r \equiv$ real, $i \equiv$ imaginary, $p \equiv$ positive, $n \equiv$ negative.

\section{Keep the hue (angle) unchanged:}

$$
f^{\prime}\left(k_{1}, k_{2}\right)=f_{r p}+j f_{i p} \pm \alpha\left(w_{r p}+j w_{i p}\right) .
$$

Our goal is to make the angle of $f^{\prime}\left(k_{1}, k_{2}\right)$ be the same as $f\left(k_{1}, k_{2}\right)$, which is $\tan ^{-1}\left(\frac{f_{i p} \pm \alpha w_{i p}}{f_{r p} \pm \alpha w_{r p}}\right)=\tan ^{-1}\left(\frac{f_{i p}}{f_{r p}}\right)$. To achieve this requirement, if $w_{r p}=p \cdot f_{r p}$ and $w_{i p}=$ $p \cdot f_{i p}$, where $\mathrm{p}$ is a constant, then $\tan ^{-1}\left(\frac{f_{i p} \pm \alpha w_{i p}}{f_{r p} \pm \alpha w_{r p}}\right)=$ $\tan ^{-1}\left(\frac{f_{i p}(1 \pm \alpha p)}{f_{r p}(1 \pm \alpha p)}\right)=\tan ^{-1}\left(\frac{f_{i p}}{f_{r p}}\right)$. Thus, the requirement is fulfilled if $\mathrm{p}=1$ :

$$
w_{r p}=f_{r p}, w_{i p}=f_{i p}
$$

\section{Make the colors of the watermarks be yellow-blue:}

$$
w_{r n}=-w_{r p}, w_{i n}=w_{i p}
$$

would do because $w_{r n}=-w_{r p}$ cancels out the real part of the watermarks, and only the imaginary part remains. As a result, the watermarks only vary along the yellow-blue axis. Substitute eq. (6) and eq. (5) into eq. (3) gives the result

$$
\begin{aligned}
& f^{\prime}\left(k_{1}, k_{2}\right)=f\left(k_{1}, k_{2}\right) \pm \alpha\left(f_{r p}+j f_{i p}\right) \\
& f^{\prime}\left(-k_{1},-k_{2}\right)=f\left(-k_{1},-k_{2}\right) \pm \alpha\left(-f_{r p}+j f_{i p}\right) .
\end{aligned}
$$

To ensure invisibility of the watermarks, we define a distortion metric $D\left(n, m, k_{1}, k_{2}\right)$ that measures the difference in the spatial domain generated by modifying the coefficients $f\left(k_{1}, k_{2}\right)$ and $f\left(-k_{1},-k_{2}\right)$ in the frequency domain, defined as:

$$
\begin{gathered}
D\left(n, m, k_{1}, k_{2}\right)=\frac{1}{N^{2}}\left(\left(f\left(k_{1}, k_{2}\right)-f^{\prime}\left(k_{1}, k_{2}\right)\right) e^{\left(\frac{2 \pi j k_{1} n}{N}\right)}\right. \\
e^{\left(\frac{2 \pi j k_{2} m}{N}\right)}+\left(f\left(-k_{1},-k_{2}\right)-f^{\prime}\left(-k_{1},-k_{2}\right)\right) e^{\left(\frac{-2 \pi j k_{1} n}{N}\right)} \\
\left.e^{\left(\frac{-2 \pi j k_{2} m}{N}\right)}\right) .
\end{gathered}
$$

Substituting eq. (7) into eq. (8) gives

$$
\begin{gathered}
D\left(n, m, k_{1}, k_{2}\right)=\frac{1}{N^{2}}\left(-\alpha\left(f_{r p}+j f_{i p}\right) e^{\left(\frac{2 \pi j k_{1} n}{N}\right)} e^{\left(\frac{2 \pi j k_{2} m}{N}\right)}\right. \\
\left.-\alpha\left(-f_{r p}+j f_{i p}\right) e^{\left(\frac{-2 \pi j k_{1} n}{N}\right)} e^{\left(\frac{-2 \pi j k_{2} m}{N}\right)}\right)
\end{gathered}
$$

If we let $\theta_{1}=\frac{2 \pi k_{1} n}{N}$, and $\theta_{2}=\frac{2 \pi k_{2} m}{N}$, with some algebraic manipulations, eq. (9) can be simplified to:

$$
\begin{gathered}
D\left(n, m, k_{1}, k_{2}\right)=\frac{1}{N^{2}}\left(-j \alpha\left(2 f_{r p}\left(\sin \theta_{2} \cos \theta_{1}+\sin \theta_{1} \cos \theta_{2}\right)\right.\right. \\
\left.\left.+2 f_{i p}\left(\cos \theta_{1} \cos \theta_{2}-\sin \theta_{1} \sin \theta_{2}\right)\right)\right)
\end{gathered}
$$


Watermark invisibility is achieved if $\left\|D\left(n, m, k_{1}, k_{2}\right)\right\|^{2} \leq$ $(N U J N C D)^{2} \forall n, m$, that is,

$$
\begin{aligned}
& \left\|D\left(n, m, k_{1}, k_{2}\right)\right\|^{2}=\frac{\alpha^{2}}{N^{4}}\left(2 f_{r p}\left(\sin \theta_{2} \cos \theta_{1}+\sin \theta_{1} \cos \theta_{2}\right)\right. \\
+ & \left.2 f_{i p}\left(\cos \theta_{1} \cos \theta_{2}-\sin \theta_{1} \sin \theta_{2}\right)\right)^{2} \leq(N U J N C D)^{2} .
\end{aligned}
$$

Depending on the perceptual requirement of the applications, we have two options to determine $\alpha$. One is to make the average distortions of a block less than $N U J N C D$ (loose condition), and the other one is to make the distortion of every pixel less than $N U J N C D$ (tight condition): Approach 1: Calculate $\alpha$ such that the average distortions of a block are less than $N U J N C D$

$$
\begin{gathered}
\left\|D\left(k_{1}, k_{2}\right)\right\|^{2}= \\
\alpha^{2} \sum_{n=0}^{N-1} \sum_{m=0}^{N-1} \frac{1}{N^{4}}\left(2 f_{r p}\left(\sin \theta_{2} \cos \theta_{1}+\sin \theta_{1} \cos \theta_{2}\right)\right. \\
\left.+2 f_{i p}\left(\cos \theta_{1} \cos \theta_{2}-\sin \theta_{1} \sin \theta_{2}\right)\right)^{2}=N^{2}(N U J N C D)^{2} .
\end{gathered}
$$

Let $\mathrm{x}=\cos \theta_{1} \cos \theta_{2}-\sin \theta_{1} \sin \theta_{2}$ and $\mathrm{y}=\sin \theta_{2} \cos \theta_{1}+$ $\sin \theta_{1} \cos \theta_{2}$,

$$
\alpha=\sqrt{\frac{N^{6}(N U J N C D)^{2}}{\sum_{n=0}^{N-1} \sum_{m=0}^{N-1}\left(2 f_{r p} y+2 f_{i p} x\right)^{2}}} .
$$

Approach 2: Recursively decrease $\alpha$ until the distortions of all pixels are smaller than $N U J N C D$. We assign an initial $\alpha$, and calculate $\left\|D\left(n, m, k_{1}, k_{2}\right)\right\|^{2}$ for every pixel of the block. If some pixel distortion exceeds $(N U J N C D)^{2}$, we decrease $\alpha$ until $\left\|D\left(n, m, k_{1}, k_{2}\right)\right\|^{2}<(N U J N C D)^{2}$ $\forall n, m$.

In general, the approach 1 should be used when the robustness of the watermarks is critical while the invisibility constraint can be relaxed. On the other hand, the approach 2 is good under the situations that the invisibility constraint is more important. To extract the embedded watermarks, we need the original image, and the watermarked (possibly corrupted) image. We first calculate $f\left(k_{1}, k_{2}\right)+\alpha\left(f_{r p}+j f_{i p}\right)$ and $f\left(k_{1}, k_{2}\right)-\alpha\left(f_{r p}+j f_{i p}\right)$ from the original image, followed by a distance comparison to the coefficient extracted from the watermarked image. If the extracted coefficient is closer to $f\left(k_{1}, k_{2}\right)+\alpha\left(f_{r p}+j f_{i p}\right)$, we assume bit 1 was embedded, otherwise, bit 0 was embedded.

\section{EXPERIMENTAL RESULTS}

We embedded up to 1 bit of information into each block imperceptibly using the above watermarking technique on
$A C(2,2)$ and $A C(6,6)$. The Lenna image with size $512 \times$ 480 was chosen for testing. We embedded 2931 bits of information into the blocks with $\alpha>10$. Figure 1 is the original image, and Figure 2 is the watermarked image using approach 1. In order to test the robustness of the algorithm, we applied some common attacks, and compared the results to two watermarking algorithms - the DCT based and AC estimation based method [10]. The PSNRs and the number of bits embedded using the SCDFT, DCT, and AC algorithms are: (35.34, 2931 bits), (34.43, 3140 bits) and $(51.89,416$ bits) respectively. The following table shows the Bit Error Rates(BERs) against histogram equalization, sharpening, and resizing.

\begin{tabular}{|c|c|c|c|}
\hline Attack & SCDFT & DCT & AC \\
\hline No attack & $0 \%$ & $0 \%$ & $0 \%$ \\
Histogram equalization & $0 \%$ & $4.30 \%$ & $4.09 \%$ \\
Sharpening & $0 \%$ & $4.71 \%$ & $4.57 \%$ \\
Resizing by factor 2 & $6.58 \%$ & $9.01 \%$ & $5.77 \%$ \\
\hline
\end{tabular}

The results for the robustness against JPEG compression and Gaussian noise are shown in Figure 3 and 4. Notice that even though the proposed algorithm embedded a large number of bits, it is still more robust to the DCT and AC estimation algorithms for most cases, except for the JPEG with low compression factor (compression factor $=$ compressed size / uncompressed size). The underlying reason is that the SCDFT algorithm only modifies the chromatic components of a color image, and JPEG quantizes them a lot for high compression, which corrupts the watermarks as the compression factor goes down.

\section{CONCLUSION}

In this paper, a new watermarking technique for digital color images has been presented. The SCDFT transform was used because the color content of a color pixel is processed holistically in the frequency domain. Two approaches using the SCDFT transform were presented for different applications, and we have also shown that the proposed algorithm is more robust against most common attacks. Future work will be devoted to embed the watermarks into multiple frequency coefficients. In addition, the algorithm proposed in this paper only marks the chromatic components, without modifying the luminance component. Our current research is looking into the interaction between the color and the luminance components of color images, and how to apply it to watermarking.

\section{REFERENCES}

[1] M. Kutter, F. Jordan, and F. Bossen, "Digital signatures of color images using amplitude modulation," in 


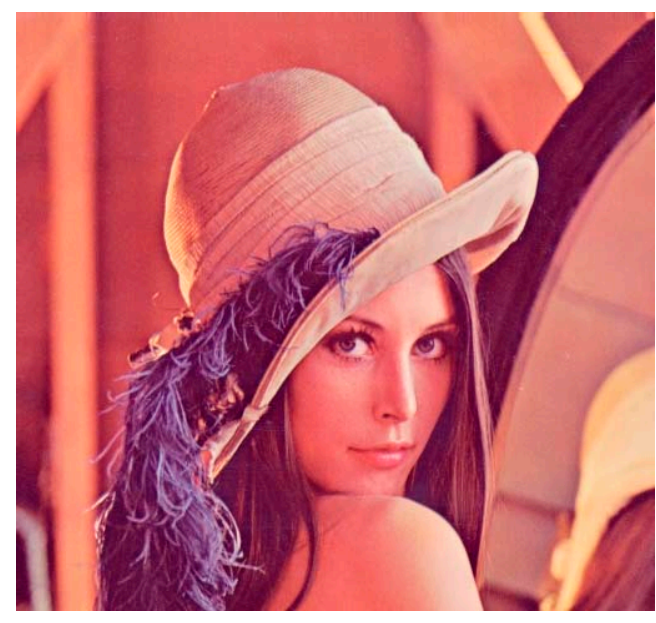

Fig. 1. Original image.

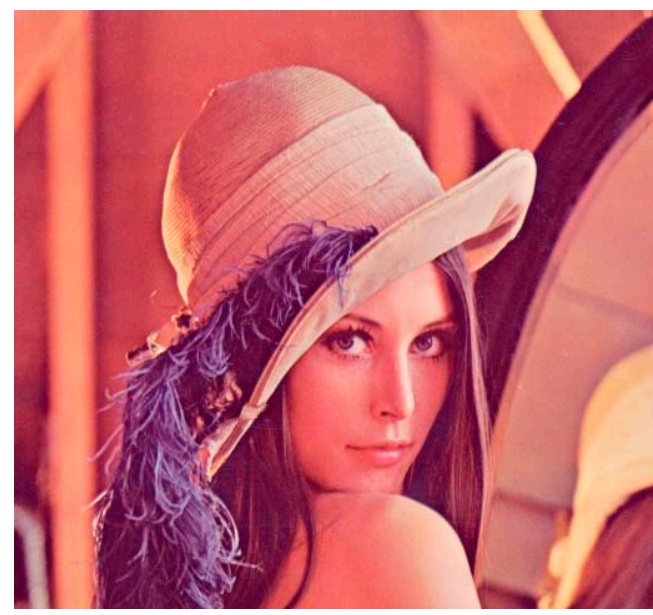

Fig. 2. Watermarked image.

SPIE, EI97 Proceedings, San Jose, California, USA, Feb. 1997, pp. 518-526.

[2] A. Piva, M. Barni, F. Bartolini, and V. Cappellini, "Exploiting the cross-correlation of rgb-channels for robust watermarking for color images" in IEEE-ICIP '99, Kobe (Japan), Oct. 1999, vol. I, pp. 306-310.

[3] A. Reed, B.Hannigan "Adaptive Color Watermarking", SPIE 2002: 222-229, April 2002.

[4] P. Bas, N. Le Bihan and J.M. Chassery, "Color watermarking using Quaternion Fourier transform", ICASSP, Hong-Kong, June 2003.

[5] Andrew McCabe and Terry Caelli, "Theory of spatiochromatic image encoding and feature extraction," $J$.

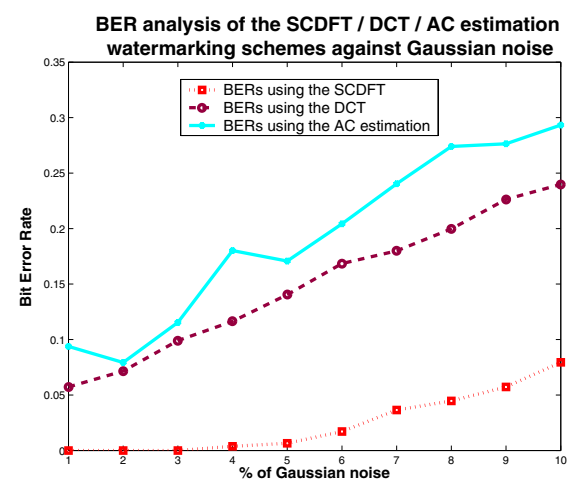

Fig. 3. BERs against Gaussian noise.

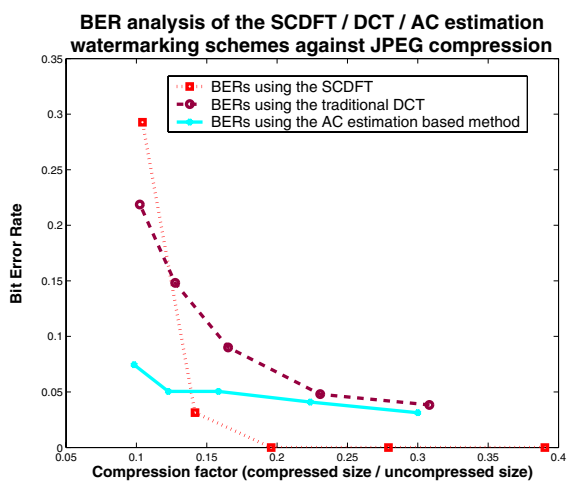

Fig. 4. BERs against JPEG compression.

of the Optical Society of America A, 17(10), 1744-1754, October 2000.

[6] Chun-Hsien Chou, Kuo-Cheng Liu, "A Visual Model for Estimating Perceptual Redundancy Inherent in Color Image". IEEE Pacific Rim Conference on Multimedia 2002: 353-360.

[7] Daniel Malacara, "Color vision and colorimetry : theory and applications", SPIE - The international Society for Optical Engineering, USA, 2002.

[8] M. Kutter and S. Winkler, "A Vision-Based Masking Model for Spread-Spectrum Image Watermarking," IEEE Trans. Image Processing, vol. 11, January 2002.

[9] Brian A. Wandell, Foundations of Vision, pp. 328. Sinauer Associates, Sunderland, Massachusetts, 1995.

[10] Y Wang and A Pearmain, "AC estimation-based Image Watermarking Method", 5th European workshop on Image Analysis and Multimedia, Lisbon, Portugal, Apr 2004 